\title{
Mindfulness and related factors among Iranian nursing students: A cross-sectional study
}

\author{
Samad Karkhah ${ }^{1,2,3}$, Ali Bazzi¹, Seyede Marzieh Adyani4, Mohammad Javad Ghazanfari5,** \\ ${ }^{1}$ Department of Medical-Surgical Nursing, School of Nursing and Midwifery, Guilan University of Medical Sciences, Rasht, Iran \\ ${ }^{2}$ Social Determinants of Health Research Center (SDHRC), Guilan University of Medical Sciences, Rasht, Iran \\ 3Burn and Regenerative Medicine Research Center, Guilan University of Medical Sciences, Rasht, Iran \\ 4Department of Medical Nanotechnology, School of Advanced Technologies in Medicine, Mazandaran University of Medical Sciences, \\ Sari, Iran \\ 5Department of Medical-Surgical Nursing, School of Nursing and Midwifery, Kashan University of Medical Sciences, Kashan, Iran
}

\begin{abstract}
Nursing students experience a number of emotional challenges during their educations, leading to high levels of stress among them. One of the strategies of stress management in nursing students is the use of mindfulness. This study aimed to assess mindfulness and related factors among Iranian nursing students. In a cross-sectional study, 100 nursing students studying at Nasibeh Faculty of Nursing and Midwifery affiliated to Mazandaran University of Medical Sciences, Iran was enrolled. Data were collected via census sampling from May to June 2018. Data were collected using a three-part questionnaire including demographic characteristics, Kentucky Mindfulness Skills scale, and Spielberger State Anxiety scale. Data analysis was performed using descriptive statistics, t-test, Chisquared, and ANOVA tests. The mean score of mindfulness of nursing students was $117.35 \pm 12.89$. The mean scores of mindfulness in the dimensions of observation, description, act with awareness, and acceptance without Judgment was $36.33 \pm 6.0,25.68 \pm 4.91,28.17 \pm 4.49$, and $27.17 \pm 3.80$, respectively. The mean score of anxiety in nursing students was $14.17 \pm 3.78$. There was a significant relationship between age and dimension "description" of mindfulness $(r=0.22, p=0.029)$. There was a significant relationship between dimension "observation" of mindfulness and experience stressful events during the last six months $(t=2.327, d f=96, p=0.022)$. Therefore, due to the importance of mindfulness among nursing students, it is possible to reduce the risk factor by creating the necessary conditions such as continuing education.
\end{abstract}

Keywords: Mindfulness, Nursing students, Anxiety, Iran

\section{Introduction}

Nursing students experience a number of emotional challenges during their educations, which lead to high levels of stress among them [1]. High levels of perceived emotional stress and anxiety cause a disorder in learning, problem-solving, and coping skills among nursing students, which ultimately hinders learning, retention, and application of knowledge in clinical and academic settings [1-3]. On the other hand, previous evidence has shown that multiple stressors such as academic education and presence in clinical settings lead to high levels of stress among nursing students [2, 4-6]. Time constraints, caring for critically ill or dying patients, fear of making

\section{"Corresponding author:}

Mohammad Javad Ghazanfari, MSc

Department of Medical-Surgical Nursing,

School of Nursing and Midwifery, Kashan University of Medical Sciences, Kashan, Iran

Tel/Fax: +98 9302426919

Email: javad.ghazanfari12@yahoo.com

(C) The Author(s) 2021

http://orcid.org/oooo-0003-3555-6044

Received: September, 20, 2021

Accepted: November, 11, 2021
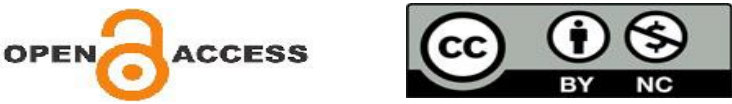
mistakes in practice, criticism and inappropriate behavior of professors, and discrimination in clinical settings are some of the other stressors for nursing students [6-9]. Finally, persistent and excessive tolerance of these stressors leads to psychological problems such as stress, anxiety, and depression [3, 10]. Based on a systematic review and meta-analysis, the prevalence of depression among nursing students was 34\% [11]. Another study in Canada showed that the prevalence of mild to severe depression, anxiety, and stress among nursing students was $33 \%, 39 \%$, and $38 \%$, respectively [12]. Increased anxiety and depression among nursing students can increase their risk of suicidal behaviors. Hence, a study in Portugal found that $5 \%$ of nursing students had suicidal behaviors [13]. A study in Iran showed that $38.7 \%$ of nursing students suffer from mild to severe depression [14].

Meanwhile, mindfulness-based training is an effective strategy for managing stress and anxiety among nursing students $[1,15-17]$. Mindfulness is defined as conscious attention to the present, a certain quality of consciousness, and attention to the moment of life. In fact, mindfulness is a clear awareness of the present reality and awareness of personal emotions $[10,18]$. Today, it is believed that conscious attention to the present increases a person's well-being [19]. Previous evidence has shown that there is a positive relationship between conscious attention to the present and factors such as negative emotions, anger, social anxiety, self-help, positive emotions, optimism, and self-esteem $[6,20]$. In addition, mindfulness can be effective in promoting emotional intelligence [21], reducing stress, anxiety, depression [22-24], reducing negative thoughts, improving sleep, increasing concentration and accuracy [2], and relaxation [25]. Thus, it is clear that effective cognition and intervention in mindfulness can help reduce stress, anxiety, and depression and improve clinical and educational skills, quality of life, well-being, empathy, and control of individual emotions among nursing students [26]. However, implementing mindfulnessbased interventions requires understanding the extent and dimensions of mindfulness.

Therefore, due to the importance of mindfulness among nursing students and limited studies in this field, the present study aimed to assess mindfulness and related factors among Iranian nursing students.

\section{Materials and Methods}

\subsection{Study design and sample}

In a cross-sectional study, 100 nursing students studying at Nasibeh Faculty of Nursing \& Midwifery affiliated to Mazandaran University of Medical Sciences, Iran was enrolled. Data were collected via census sampling from May to June 2018. Nursing students from 2 to 6 semesters were included in the present study. Students of 1 (due to lack of familiarity with the concept of mindfulness), 7 and 8 semesters (due to lack of access to them), and participants who were not satisfied were excluded from this study.

\subsection{Data collection}

Data were collected using a three-part questionnaire including demographic characteristics, Kentucky mindfulness skills (KIMS) scale, and Spielberger state anxiety scale.

\subsubsection{Demographic characteristics}

Demographic characteristics of nursing students such as age, sex, place of residence, marital status, semester, grade point average, experience stressful events during the last six months, smoking, perceived academic difficulty, the economic situation, satisfaction from their performance, and perceived communication problems were collected.

\subsubsection{Kentucky inventory of mindfulness skills}

This 39-item questionnaire was designed by Beer et al. (2004) to assess four components of mindfulness including observing (12 items), describing (8 items), acting with awareness (10 items), and accepting without judgment (9items). Participants responded to the items in this questionnaire using a five-point Likert scale from never (score of 1) to always (score of 5) [27]. This scale is scored from 39 to 195. In Iran, this tool was confirmed by Narimani et al. with Cronbach's alpha of 0.82 [28]. scale

\subsubsection{A short form of Spielberger state anxiety}

This 6-item questionnaire assesses levels of anxiety. Participants responded to the items in this questionnaire using a four-point Likert scale from at all (score of 1 ) to many (score of 4). This scale is scored from 6 to 24. This scale assesses anxiety at three levels: 1) mild (6 to 11), 2) moderate (12 to 17), and 3) severe 
(18 to 24). In a similar study, the correlation coefficient between the full form and the shortened form of the instrument was 96\% [29].

\subsection{Ethical consideration}

This research was approved by the ethics committee of Mazandaran University of Medical Sciences. The objectives of the present study were explained to the participants and their informed consent was obtained. Questionnaires were completed by nursing students, independently. The questionnaires were anonymous to ensure the confidentiality of the information.

\subsection{Data analysis}

SPSS for Windows, version 16.0 (SPSS Inc., Chicago, IL, USA) was used for statistical analysis. Continuous and categorical variables were presented using mean (standard deviation) and frequency (percentage). The Shapiro-Wilk test was used to assess the normality of data distribution. Due to the normal distribution of data, $t$-test and ANOVA were used to assess differences between the groups. Also, the Chisquare test was used to assess the relationship between nursing students' mindfulness and study variables. Statistical significance was considered $\mathrm{P}<0.05$.

\section{Results}

3.1 Participants characteristics

A total of 100 nursing students were included in the present study. Of the participants, $56 \%$ were female, $71 \%$ were single, $75 \%$ were indigenous, $10 \%$ were smokers, $37 \%$ were in good financial condition, $27 \%$ had experienced stressful events during the past six months, and $44 \%$ had communication problems. The mean age and grade of nursing students were 21.8 \pm 2.6 and $16.42 \pm 1.40$, respectively.

3.2 Mindfulness and related factors among nursing students

The mean score of mindfulness of nursing students was $117.35 \pm 12.89$. The mean scores of mindfulness in the dimensions of observation, description, act with awareness, and acceptance without Judgment was $36.33 \pm 6.0,25.68 \pm 4.91,28.17$ \pm 4.49 , and $27.17 \pm 3.80$, respectively (Table 1 ). The mean score of anxiety in nursing students was $14.17 \pm$ 3.78. There was a significant relationship between age and dimension "description" of mindfulness $(\mathrm{r}=0.22$, $\mathrm{p}=0.029$ ).

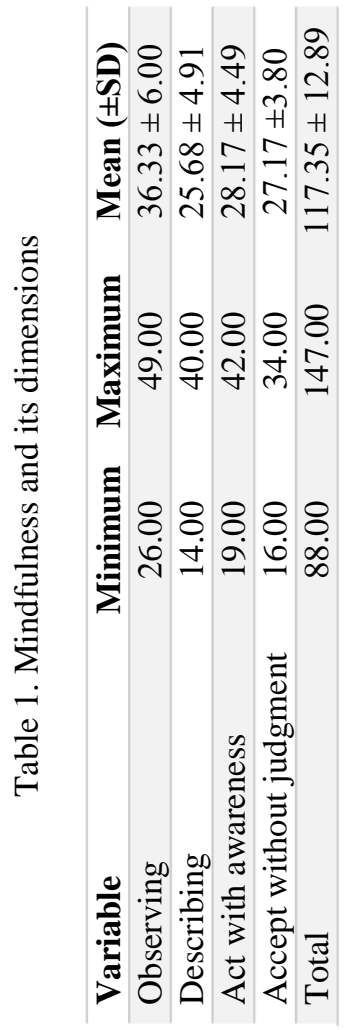

The mean score of mindfulness was higher in women compared to men ( $p>0.05)$. The mean scores of "act with awareness" and "acceptance without judgment" were higher in women compared to men $(\mathrm{t}$ $=0.239, \mathrm{df}=98, \mathrm{p}=0.811)$. The mean scores of "observation" and "description" dimensions of mindfulness were higher in men compared to women ( $>$ > 0.05).

The mean scores of mindfulness in the dimensions of "observation", "act with awareness", and "acceptance without judgment" were higher in single students compared to married students ( $p>$ 0.05). However, married students in the "descriptive" dimension of mindfulness had higher scores than single students ( $p>0.05)$.

As shown in Table 2, there was no significant relationship between mindfulness and experience stressful events during the last six months $(\mathrm{p}=0.295)$. However, there was a significant relationship between dimension "observation" of mindfulness and 
Table 2. The relationship between mindfulness and demographic characteristics among nursing students $(\mathrm{N}=100)$

\begin{tabular}{|c|c|c|c|c|}
\hline Variable & Mean \pm SD & DF & t-test & $P$ value \\
\hline \multicolumn{5}{|l|}{ Sex } \\
\hline Male & $117.00 \pm 14.39$ & \multirow[t]{2}{*}{98} & \multirow[t]{2}{*}{0.239} & \multirow[t]{2}{*}{0.811} \\
\hline Female & $117.62 \pm 11.70$ & & & \\
\hline \multicolumn{5}{|l|}{ Marital status } \\
\hline Single & $117.84 \pm 12.99$ & \multirow[t]{2}{*}{98} & \multirow[t]{2}{*}{0.599} & \multirow[t]{2}{*}{0.551} \\
\hline Married & $116.13 \pm 12.80$ & & & \\
\hline \multicolumn{5}{|c|}{ Place of residence } \\
\hline Dorm & $115.32 \pm 10.96$ & \multirow[t]{2}{*}{98} & \multirow[t]{2}{*}{0.814} & \multirow[t]{2}{*}{0.418} \\
\hline With family & $117.97 \pm 13.56$ & & & \\
\hline \multicolumn{5}{|l|}{ Smoker } \\
\hline Yes & $112.50 \pm 12.91$ & \multirow[t]{2}{*}{98} & \multirow[t]{2}{*}{-1.257} & \multirow[t]{2}{*}{0.212} \\
\hline No & $117.88 \pm 12.85$ & & & \\
\hline \multicolumn{5}{|c|}{ Experience stressful events during the last six months } \\
\hline Yes & $119.70 \pm 13.64$ & \multirow[t]{2}{*}{96} & \multirow[t]{2}{*}{1.052} & \multirow[t]{2}{*}{0.295} \\
\hline No & $116.61 \pm 12.69$ & & & \\
\hline \multicolumn{5}{|c|}{ Communication problem } \\
\hline Yes & $116.68 \pm 12.55$ & \multirow[t]{2}{*}{98} & \multirow[t]{2}{*}{-0.457} & \multirow[t]{2}{*}{0.648} \\
\hline No & $117.87 \pm 13.25$ & & & \\
\hline
\end{tabular}

experience stressful events during the last six months $(\mathrm{t}=\mathbf{2 . 3 2 7}, \mathrm{df}=96, \mathrm{p}=0.022)$.

There was no significant relationship between mindfulness and communication problems $(\mathrm{p}=$ o.648). However, there was a significant relationship between the "observation" and "description" dimensions of mindfulness and communication problems $(\mathrm{t}=\mathbf{2 . 1 8 5}$, $\mathrm{df}=98, \mathrm{p}=0.031)$.

There was no significant relationship between mindfulness and smoking $(\mathrm{p}=0.212)$. However, the mean score of the "act with awareness" dimension of mindfulness was higher in non-smokers compared to smokers ( $p$ <0.05). Also, non-smokers had less anxiety compared to smokers ( $\mathrm{p}<0.05)$.

There was no significant difference between mindfulness scores and anxiety levels $(\mathrm{p}=0.4)$. However, there was a significant relationship between levels of anxiety and the "accept without judgment" dimension of mindfulness ( $\mathrm{p}=0.043)$. Scheffe's Post Hoc test showed that "accept without judgment" reduces anxiety levels from mild to severe.

\section{Discussion}

The findings of the present study showed that nursing students had a high level of mindfulness. Inconsistent with this finding, a study in the USA found that nursing students had a moderate to low level of mindfulness [2]. This discrepancy may be due to differences in organizational contexts and academic settings. On the other hand, the level of mindfulness of last semester students is lower compared to firstsemester students, which can be due to higher expectations, focus on goals, and advances in clinical education [30]. In this regard, first semester students have attention deficit disorder due to problems such as stress, distraction, and high memory impairment which can be one of the reasons for a low level of mindfulness in them [31]. Nursing educators have an important role to play in reducing these problems for nursing students. Identifying the causes of these problems in students and implementing effective interventions can reduce psychological distress such as stress, anxiety, and depression in nursing students [32]. Hence, the findings of a systematic review and meta-analysis showed that mindfulness-based interventions can play an important role in reducing anxiety, depression, and distraction in nursing students [32].

Based on the findings of this study, nursing students with communication problems had a low level of mindfulness. This finding was consistent with the results of studies in the USA, Norway, and Canada [33-35]. Nursing students with communication problems have behavioral problems such as 
inattention to others, impulsivity, and negative personality traits, which can lead to a low level of mindfulness [35]. Consistent with this finding, a study in the USA found that there was a positive association between mindfulness and the tendency to communicate [36].

As the present study showed, there was a significant relationship between age and dimension "description" of mindfulness. This finding was consistent with the results of a study in the USA [37]. However, another study in the USA showed that there is no significant relationship between age and mindfulness [38]. These differences may be due to differences in personality traits and social and educational desirability in different societies [39].

In this study, the mean score of mindfulness was higher in women compared to men, which was confirmed by a study in Iran [40]. Inconsistent with this finding, a study in the USA found that mindfulness was higher in men than in women [41]. These contradictions can be due to differences in individual characteristics, cultural and social conditions [42], and religious conflicts and tendencies in different societies [43]. However, these variables can affect people's mental health and ultimately affect mindfulness. Therefore, it is recommended that well-designed studies in the future be conducted in this area.

In the present study, half of the nursing students suffer from academic stress. Academic stress can be due to the variety of curricula and different educational policies in different faculties that affect the level of mindfulness of nursing students [40]. However, identifying different educational aspects such as new educational ideas, playing a role in presenting lesson plans, and creating diversity in the classroom can potentially be effective in improving the mindfulness of nursing students [44]. Nursing students face higher stress compared to students in other disciplines. Hence, academic stress leads to adverse outcomes for physical and mental health, absenteeism, and poor academic performance in nursing students [45]. Therefore, it is suggested that nursing educators provide coping strategies for nursing students to improve resilience and reduce perceived stress.

In the present study, there was no significant difference between mindfulness scores and anxiety levels. However, there was a significant relationship between levels of anxiety and the "accept without judgment" dimension of mindfulness. Also, "accept without judgment" reduces anxiety levels from mild to severe. Inconsistent with this finding, a study in Iran showed that there was a significant and negative relationship between mindfulness and anxiety [46]. Another study in Iran showed that a mindfulnessbased stress reduction program (MBSR) was effective in improving students' mindfulness, expression, and anxiety [29]. However, previous evidence in the United Kingdom [47], South Korea [16], USA [48], Portugal, and Brazil [49] have shown that MBSR is effective in reducing stress, anxiety, and depression among nursing students. Obviously, MBSR can be used as a promising way to reduce stress in nursing students.

This study had several limitations. The main limitation of the present study was the lack of generalizability of the findings to all nursing students due to the limited sample size. Also, the self-report nature of the questionnaires may not adequately reflect mindfulness.

Overall, the findings of the present study showed that the mindfulness of nursing students was at a high level. Therefore, due to the importance of mindfulness among nursing students and its relationship with variables such as age, marital status, the experience of stressful events, communication problems, and anxiety, it is possible to reduce the risk factor by creating the necessary conditions such as continuing education. Due to the importance of mindfulness among nursing students, it is recommended that more attention be paid to evidence-based interventions in this area. Also, considering a mind-based program in nursing students' curriculum as a way to improve work memory capacity and ensure success in emotion regulation is inevitable. It is recommended that future studies assess mindfulness, emotion regulation, and working memory capacity among nursing students. Nursing students typically experience high levels of stress in educational and clinical settings. This stress leads them to anxiety or depression and reduced academic performance. Meanwhile, nursing educators must prioritize student health. Also, nursing policymakers and managers can play an important role by including mindfulness programs in nursing students' courses and holding workshops in this field. 


\section{Author contributions}

All authors contributed equally in data collection and drafting of the manuscript. Also, all authors approved the final version of the manuscript.

\section{Conflict of interests}

The authors declare that they have no conflict of interest.

\section{Ethical declarations}

This research was approved by the ethics committee of Mazandaran University of Medical Sciences.

\section{Financial Support}

This research did not receive any specific grant from funding agencies in the public, commercial, or not-for-profit sectors.

\section{References}

1. Lu J, Mumba MN, Lynch S, Li C, Hua C, Allen RS. Nursing students' trait mindfulness and psychological stress: A correlation and mediation analysis. Nurse Educ Today. 2019; 75:41-6.

2. Dubert CJ, Schumacher AM, Locker L, Gutierrez AP, Barnes VA. Mindfulness and emotion regulation among nursing students: Investigating the mediation effect of working memory capacity. Mindfulness. 2016; 7(5):1061-70.

3. Burger KG, Lockhart JS. Meditation's Effect on Attentional Efficiency, Stress, and Mindfulness Characteristics of Nursing Students. J Nurs Educ. 2017; 56(7):430-4.

4. Ching SSY, Cheung K, Hegney D, Rees CS. Stressors and coping of nursing students in clinical placement: A qualitative study contextualizing their resilience and burnout. Nurse Educ Pract. 2020; 42:102690.

5. Beanlands H, McCay E, Fredericks S, Newman K, Rose D, Santa Mina E, et al. Decreasing stress and supporting emotional wellbeing among senior nursing students: A pilot test of an evidencebased intervention. Nurse Educ Today. 2019; 76:222-7.

6. Yüksel A, Bahadır Yllmaz E. The effects of group mindfulnessbased cognitive therapy in nursing students: A quasi-experimental study. Nurse Educ Today. 2020; 85:104268.

7. Brown K, Anderson-Johnson P, McPherson AN. Academicrelated stress among graduate students in nursing in a Jamaican school of nursing. Nurse Educ Pract. 2016; 20:117-24.

8. Labrague LJ, McEnroe-Petitte DM, De Los Santos JAA, Edet OB. Examining stress perceptions and coping strategies among Saudi nursing students: A systematic review. Nurse Educ Today. 2018; 65:192-200.

9. Aliafsari Mamaghani E, Rahmani A, Hassankhani H, Zamanzadeh V, Campbell S, Fast O, et al. Experiences of Iranian Nursing Students Regarding Their Clinical Learning Environment. Asian Nurs Res (Korean Soc Nurs Sci). 2018; 12(3):216-22.
10. Ratanasiripong P, Park JF, Ratanasiripong N, Kathalae D. Stress and Anxiety Management in Nursing Students: Biofeedback and Mindfulness Meditation. J Nurs Educ. 2015; 54(9):520-4. 11. Tung YJ, Lo KKH, Ho RCM, Tam WSW. Prevalence of depression among nursing students: A systematic review and meta-analysis. Nurse Educ Today. 2018; 63:119-29.

12. Chernomas WM, Shapiro C. Stress, depression, and anxiety among undergraduate nursing students. Int $J$ Nurs Educ Scholarsh. 2013; 10: 255-266.

13. Leal SC, Santos JC. Suicidal behaviors, social support and reasons for living among nursing students. Nurse Educ Today. 2016; 36:434-8.

14. Rezayat F, Dehghan Nayeri N. The Level of Depression and Assertiveness among Nursing Students. Int J Community Based Nurs Midwifery. 2014; 2(3):177-84.

15. Ghawadra SF, Abdullah KL. Mindfulness-based stress reduction for psychological distress among nurses: A systematic review. J Clin Nurs. 2019; 28(21-22):3747-58.

16. Song Y, Lindquist R. Effects of mindfulness-based stress reduction on depression, anxiety, stress and mindfulness in Korean nursing students. Nurse Educ Today. 2015; 35(1):86-90.

17. van der Riet $P$, Levett-Jones $T$, Aquino-Russell C. The effectiveness of mindfulness meditation for nurses and nursing students: An integrated literature review. Nurse Educ Today. 2018; 65:201-11.

18. Arthur D, Dizon D, Jooste K, Li Z, Salvador M, Yao X. Mindfulness in nursing students: The five facet mindfulness questionnaire in samples of nursing students in China, the Philippines, and South Africa. Int J Ment Health Nurs. 2018; 27(3): $975-86$.

19. Pérula-de Torres LA, Atalaya JCV, García-Campayo J, RoldánVillalobos A, Magallón-Botaya R, Bartolomé-Moreno C, et al. Controlled clinical trial comparing the effectiveness of a mindfulness and self-compassion 4-session programme versus an 8-session programme to reduce work stress and burnout in family and community medicine physicians and nurses: MINDUUDD study protocol. BMC Fam Pract. 2019; 20(1):24.

20. Gracia-Gracia P, Oliván-Blázquez B. Burnout and Mindfulness Self-Compassion in Nurses of Intensive Care Units: CrossSectional Study. Holist Nurs Pract. 2017; 31(4):225-33.

21. Snowden A, Stenhouse R, Young J, Carver H, Carver F, Brown $\mathrm{N}$. The relationship between emotional intelligence, previous caring experience and mindfulness in student nurses and midwives: a cross sectional analysis. Nurse Educ Today. 2015; 35(1):152-8.

22. Burns JL, Lee RM, Brown LJ. The effect of meditation on selfreported measures of stress, anxiety, depression, and perfectionism in a college population. J College Stud Psychother. 2011; 25(2):132-44.

23. Hall CW, Row KA, Wuensch KL, Godley KR. The role of selfcompassion in physical and psychological well-being. J Psychol. 2013; 147(4):311-23.

24. Terry ML, Leary MR, Mehta S. Self-compassion as a buffer against homesickness, depression, and dissatisfaction in the transition to college. Self Identity. 2013; 12(3):278-90.

25. Sanko J, McKay M, Rogers S. Exploring the impact of mindfulness meditation training in pre-licensure and post graduate nurses. Nurse Educ Today. 2016; 45:142-7. 


\section{Karkhah et al.}

26. Kinsella EA, Smith K, Bhanji S, Shepley R, Modor A, Bertrim A. Mindfulness in allied health and social care professional education: a scoping review. Disabil Rehabil. 2020; 42(2):283-95. 27. Baer RA, Smith GT, Allen KB. Assessment of mindfulness by self-report: the Kentucky inventory of mindfulness skills. Assessment. 2004; 11(3):191-206.

28. Narimani M, Zahed A, Golpour R. The relationship of mindfulness coping styles and emotional intelligence with mental health among the students in Payame-Noor university in Mazandaran. J Instr Evaluat. 2012; 5(19):91-105.

29. Golpour Chamarkohi R, Mohammadamini Z. The efficacy of mindfulness based stress reduction on mindfulness and assertiveness of students with test anxiety. J Sch Psychol. 2012; 1(3):82-100.

30. Lynch S, Gander ML, Kohls N, Kudielka B, Walach H. Mindfulness-based coping with university life: A non-randomized wait-list-controlled pilot evaluation. Stress Health. 2011; 27(5):365-75.

31. Jha AP, Stanley EA, Kiyonaga A, Wong L, Gelfand L. Examining the protective effects of mindfulness training on working memory capacity and affective experience. Emotion. 2010; 10(1):54-64.

32. Li Y-F, Sun W-X, Sun X-J, Sun J, Yang D-M, Jia B-L, et al. Effects of mindfulness meditation on anxiety, depression, stress, and mindfulness in nursing students: A meta-analysis and trial sequential analysis of randomized controlled trials. Front Nurs. 2020; 7(1):59-69.

33. Brown KW, Ryan RM. The benefits of being present: mindfulness and its role in psychological well-being. J Pers Soc Psychol. 2003; 84(4):822-48.

34. Thompson BL, Waltz J. Everyday mindfulness and mindfulness meditation: Overlapping constructs or not? Pers Individ Dif. 2007; 43(7):1875-85.

35. Sundling V, Sundler AJ, Holmström IK, Kristensen DV, Eide $\mathrm{H}$. Mindfulness predicts student nurses' communication selfefficacy: A cross-national comparative study. Patient Educ Couns. 2017; 100(8):1558-63.

36. Sweet DM, Gentile D, He L. Communication Apprehension and Willingness to Communicate in Veterinary Medicine Students: Implications for Mindfulness and Communication Training. Health Commun. 2021:1-9.

37. Baer RA, Smith GT, Lykins E, Button D, Krietemeyer J, Sauer $\mathrm{S}$, et al. Construct validity of the five facet mindfulness questionnaire in meditating and nonmeditating samples. Assessment. 2008; 15(3):329-42.

38. Lykins EL, Baer RA. Psychological functioning in a sample of long-term practitioners of mindfulness meditation. $\mathrm{J}$ Cogn Psychother. 2009; 23(3):226-41.

39. Munif B, Poeranto S, Utami YW. Effects of islamic spiritual mindfulness on stress among nursing students. Nurse Media $\mathrm{J}$ Nurs. 2019; 9(1):69-77.

40. Ying C, Liu CJ, He J, Wang J. Academic stress and evaluation of a mindfulness training intervention program. NeuroQuantology. 2018; 16(5): 97-103.

41. Creswell JD, Way BM, Eisenberger NI, Lieberman MD. Neural correlates of dispositional mindfulness during affect labeling. Psychosom Med. 2007; 69(6):560-5.
42. Vonderlin R, Biermann M, Bohus M, Lyssenko L. Mindfulnessbased programs in the workplace: a meta-analysis of randomized controlled trials. Mindfulness. 2020; 11(7):1579-98.

43. Walker AR. "God is my doctor": mindfulness meditation/prayer as a spiritual well-being coping strategy for Jamaican school principals to manage their work-related stress and anxiety. J Educ Adm. 2020; 58(4):467-80.

44. Lee M, Jang KS. Nursing Students' Meditative and Sociocognitive Mindfulness, Achievement Emotions, and Academic Outcomes: Mediating Effects of Emotions. Nurse Educ. 2021; 46(3):E39-E44.

45. Narimani M, Amini M, Abolghasemi A, Zahed Babolan A. Comparison of Mindfulness and Anxiety Sensitivity among Students with Compulsive Hoarding and Body Dysmorphic. J Clin Psychol. 2013; 5(3):23-33.

46. Aeamla-Or N. The effect of mindfulness-based stress reduction on stress, depression, self-esteem and mindfulness in Thai nursing students: A randomised controlled trial. Doctoral disseratation, University of Newcastle, Newcastle. 2015. Available from: https://nova.newcastle.edu.au/vital/access/manager/Repository /uon:22445

47. Morrison Wylde C, Mahrer NE, Meyer RML, Gold JI. Mindfulness for Novice Pediatric Nurses: Smartphone Application Versus Traditional Intervention. J Pediatr Nurs. 2017; 36:205-12. 48. Duarte J, Pinto-Gouveia J. Mindfulness, self-compassion and psychological inflexibility mediate the effects of a mindfulnessbased intervention in a sample of oncology nurses. J Contextual Behav Sci. 2017; 6(2):125-33.

49. dos Santos TM, Kozasa EH, Carmagnani IS, Tanaka LH, Lacerda SS, Nogueira-Martins LA. Positive Effects of a Stress Reduction Program Based on Mindfulness Meditation in Brazilian Nursing Professionals: Qualitative and Quantitative Evaluation. Explore (NY). 2016; 12(2):90-9. 